Research Article

\title{
Spacelike Hypersurfaces in Weighted Generalized Robertson-Walker Space-Times
}

\author{
Ximin Liu (D) and Ning Zhang (D) \\ School of Mathematical Sciences, Dalian University of Technology, Dalian 116024, China \\ Correspondence should be addressed to Ximin Liu; ximinliu@dlut.edu.cn
}

Received 28 October 2017; Accepted 6 February 2018; Published 5 March 2018

Academic Editor: Remi Léandre

Copyright (c) 2018 Ximin Liu and Ning Zhang. This is an open access article distributed under the Creative Commons Attribution License, which permits unrestricted use, distribution, and reproduction in any medium, provided the original work is properly cited.

Applying generalized maximum principle and weak maximum principle, we obtain several uniqueness results for spacelike hypersurfaces immersed in a weighted generalized Robertson-Walker (GRW) space-time under suitable geometric assumptions. Furthermore, we also study the special case when the ambient space is static and provide some results by using Bochner's formula.

\section{Introduction}

In recent years, spacelike hypersurfaces in Lorentzian manifolds have been deeply studied not only from their mathematical interest, but also from their importance in general relativity.

Particularly, there are many articles that study spacelike hypersurfaces in weighted warped product space-times. A weighted manifold is a Riemannian manifold with a measure that has a smooth positive density with respect to the Riemannian one. More precisely, the weighted manifold $M_{f}$ associated with a complete $n$-dimensional Riemannian manifold $\left(M^{n}, g\right)$ and a smooth function $f$ on $M^{n}$ is the triple $\left(M^{n}, g, d \mu=e^{-f} d M\right)$, where $d M$ stands for the volume element of $M^{n}$. In this setting, we will take into account the so-called Bakry-Émery Ricci tensor (see [1]) which as an extension of the standard Ricci tensor Ric, which is defined by

$$
\operatorname{Ric}_{f}=\text { Ric }+ \text { Hess } f .
$$

Therefore, it is natural to extend some results of the Ricci curvature to analogous results for the Bakry-Émery Ricci tensor. Before giving more details on our work we present a brief outline of some recent results related to our one.

In [2], Wei and Wylie considered the complete $n$-dimensional weighted Riemannian manifold and proved mean curvature and volume comparison results on the assumption that the $\infty$-Bakry-Émery Ricci tensor is bounded from below and $f$ or $|\nabla f|$ is bounded. Later, Cavalcante et al. [3] researched the Bernstein-type properties concerning complete twosided hypersurfaces immersed in a weighted warped product space using the appropriated generalized maximum principles. Moreover, [4] obtained new Calabi-Bernstein's type results related to complete spacelike hypersurfaces in a weighted GRW space-time. More recently, some rigidity results of complete spacelike hypersurfaces immersed into a weighted static GRW space-time are given in [5].

In this paper we study spacelike hypersurfaces in a weighted generalized Robertson-Walker (GRW) space-times. Moreover, a GRW space-time is a space-time regarding a warped product of a negative definite interval as a base, a Riemannian manifold as a fiber, and a positive smooth function as a warped function. Furthermore, there exists a distinguished family of spacelike hypersurfaces in a GRW space-time, that is, the so-called slices, which are defined as level hypersurfaces of the time coordinate of the space-time. Notice that any slice is totally umbilical and has constant mean curvature.

We have organized this paper as follows. In Section 2, we introduce some basic notions to be used for spacelike hypersurfaces immersed in weighted GRW space-times. In Section 3, we prove some uniqueness results of spacelike hypersurface in a weighted GRW space-time under appropriate conditions on the weighted mean curvature and the 
weighted function by using the generalized Omori-Yau maximum principle or the weak maximum principle. Finally, in Section 4, applying the weak maximum principle, we obtain some rigidity results for the special case when the ambient space is static.

\section{Preliminaries}

Let $M^{n}$ be a connected $n$-dimensional oriented Riemannian manifold and $I$ be an open interval in $\mathbb{R}$ endowed with the metric $-d t^{2}$. We let $\rho: I \rightarrow \mathbb{R}^{+}$be a positive smooth function. Denote $-I \times{ }_{\rho} M^{n}$ to be the warped product endowed with the Lorentzian metric

$$
\langle,\rangle=-\pi_{I}^{*}\left(d t^{2}\right)+\rho\left(\pi_{I}\right)^{2} \pi_{M}^{*}\left(\langle,\rangle_{M}\right),
$$

where $\pi_{I}$ and $\pi_{M}$ are the projections onto $I$ and $M$, respectively. This space-time is a warped product in the sense of ([6], Chap. 7), with fiber $(M,\langle\rangle$,$) , base \left(I,-d t^{2}\right)$, and warping function $\rho$. Furthermore, for a fixed point $t_{0} \in I$, we say that $M_{t_{0}}^{n}=-\left\{t_{0}\right\} \times M^{n}$ is a slice of $-I \times{ }_{\rho} M^{n}$. Following the terminology used in [7], we will refer to $-I \times{ }_{\rho} M^{n}$ as a generalized Robertson-Walker (GRW) space-time. Particularly, if the fiber $M^{n}$ has constant section curvature, it is called a Robertson-Walker $(R W)$ space-time.

Recall that a smooth immersion $\psi: \Sigma^{n} \rightarrow-I \times{ }_{\rho} M^{n}$ of an $n$-dimensional connected manifold $\Sigma^{n}$ is called a spacelike hypersurface if the induced metric via $\psi$ is a Riemannian metric on $\Sigma^{n}$, which will be also denoted for $\langle$,$\rangle .$

In the following, we will deal with two particular functions naturally attached to spacelike hypersurface $\Sigma^{n}$, namely, the angle (or support) function $\Theta=\left\langle N, \partial_{t}\right\rangle$ and the height function $h=\left.\left(\pi_{I}\right)\right|_{\Sigma}$, where $\partial_{t}:=\partial / \partial t$ is a (unitary) timelike vector field globally defined on $\bar{M}$ and $N$ is a unitary timelike normal vector field globally defined on $\Sigma$.

Let $\bar{\nabla}$ and $\nabla$ stand for gradients with respect to the metrics of $-I \times{ }_{\rho} M^{n}$ and $\Sigma^{n}$, respectively. By a simple computation, we have

$$
\bar{\nabla} \pi_{I}=-\left\langle\bar{\nabla} \pi_{I}, \partial_{t}\right\rangle \partial_{t}=-\partial_{t}
$$

Therefore, the gradient of $h$ on $\Sigma^{n}$ is

$$
\nabla h=\left(\bar{\nabla} \pi_{I}\right)^{\top}=-\partial_{t}^{\top}=-\partial_{t}-\Theta N .
$$

Particularly, we have

$$
|\nabla h|^{2}=-1+\Theta^{2}
$$

where || denotes the norm of a vector field on $\Sigma^{n}$.

Now, we consider that a GRW space-time $-I \times_{\rho} M^{n}$ is endowed with a weighted function $f$, which will be called a weighted GRW space-time $-I \times{ }_{\rho} M_{f}^{n}$. In this setting, for a spacelike hypersurface $\Sigma^{n}$ immersed into $-I \times{ }_{\rho} M_{f}^{n}$, the $f$ divergence operator on $\Sigma^{n}$ is defined by

$$
\operatorname{div}_{f}(X)=e^{f} \operatorname{div}\left(e^{-f} X\right)
$$

where $X$ is a tangent vector field on $\Sigma^{n}$.
For a smooth function $u: \Sigma^{n} \rightarrow \mathbb{R}$, we define its drifting Laplacian by

$$
\Delta_{f} u=\operatorname{div}_{f}(\nabla u)=\Delta u-\langle\nabla u, \nabla f\rangle,
$$

and we will also denote such an operator as the $f$-Laplacian of $\Sigma^{n}$.

According to Gromov [8], the weighted mean curvature or $f$-mean curvature $H_{f}$ of $\Sigma^{n}$ is given by

$$
n H_{f}=n H-\langle\bar{\nabla} f, N\rangle \text {, }
$$

where $H$ is the standard mean curvature of hypersurface $\Sigma^{n}$ with respect to the Gauss map $N$.

It follows from a splitting theorem due to Case (see [9] Theorem 1.2) that if a weighted GRW space-time $-I \times{ }_{\rho} M_{f}^{n}$ is endowed with a bounded weighted function $f$ such that $\overline{\operatorname{Ric}}_{f}(V, V) \geq 0$ for all timelike vector fields $V$ on $-I \times_{\rho} M_{f}^{n}$, then $f$ must be constant along $\mathbb{R}$. In the same spirit of this result, in the following we will consider weighted GRW spacetimes $-I \times_{\rho} M_{f}^{n}$ whose weighted function $f$ does not depend on the parameter $t \in I$; that is, $\left\langle\bar{\nabla} f, \partial_{t}\right\rangle=0$. Moreover, for simplicity, we will refer to them as $\bar{M}^{n+1}:=-I \times{ }_{\rho} M_{f}^{n}$.

In the following, we give some technical lemmas that will be essential for the proofs of our main results in weighted GRW space-times $\bar{M}^{n+1}=-I \times{ }_{\rho} M_{f}^{n}$ (for further details on the proof, see Lemma 1 in [4]).

Lemma 1. Let $\Sigma^{n}$ be a spacelike hypersurface immersed in a weighted GRW spacetime $\bar{M}^{n+1}=-I \times{ }_{\rho} M_{f}^{n}$, with height function $h$. Then,

$$
\begin{aligned}
\Delta_{f} h= & -(\log \rho)^{\prime}(h)\left(n+|\nabla h|^{2}\right)-n H_{f} \Theta, \\
\Delta_{f} \rho(h)= & -n \frac{\rho^{\prime}(h)^{2}}{\rho(h)}+|\nabla h|^{2} \rho(h)(\log \rho)^{\prime \prime}(h) \\
& -n \rho^{\prime}(h) H_{f} \Theta .
\end{aligned}
$$

If we denote $\mathscr{L}_{f}^{1}$ as the space of the integrable functions on $\Sigma^{n}$ with respect to the weighted volume element $d \mu=$ $e^{-f} d \Sigma$, using the relation of $\operatorname{div}_{f}(X)=e^{f} \operatorname{div}\left(e^{-f} X\right)$ and Proposition 2.1 in [10], we can obtain the following extension of a result in [11].

Lemma 2. Let $u$ be a smooth function on a complete weighted Riemannian manifold $\Sigma^{n}$ with weighted function $f$ such that $\Delta_{f} u$ does not change sign on $\Sigma^{n}$. If $|\nabla u| \in \mathscr{L}_{f}^{1}$, then $\Delta_{f} u$ vanishes identically on $\Sigma^{n}$.

In the following, we will introduce the weak maximum principle for the drifted Laplacian. By the fact in [12], that is, the Riemannian manifold $M$ satisfies the weak maximum principle if and only if $M$ is stochastically complete, we can have the next lemma which extended a result of [13]. 
Lemma 3. Let $\left(M^{n},\langle\rangle,, e^{-f} d M\right)$ be ann-dimensional stochastically complete weighted Riemannian manifold and $u: M \rightarrow$ $\mathbb{R}$ be a smooth function which is bounded from below on $M^{n}$. Then there is a sequence of points $p_{k} \in M^{n}$ such that

$$
\begin{aligned}
\lim _{k} u\left(p_{k}\right) & =\inf u, \\
\lim _{k} \Delta_{f} u\left(p_{k}\right) & \geq 0 .
\end{aligned}
$$

Equivalently, for any smooth function $u: \Sigma^{n} \rightarrow \mathbb{R}$ which is bounded from above on $M^{n}$, there is a sequence of points $q_{k} \in$ $M^{n}$ such that

$$
\begin{aligned}
\lim _{k} u\left(q_{k}\right) & =\sup u, \\
\lim _{k} \Delta_{f} u\left(q_{k}\right) & \leq 0 .
\end{aligned}
$$

\section{Uniqueness Results in Weighted GRW Space-Times}

In this section, we will state and prove our main results in weighted GRW space-times $\bar{M}^{n+1}=-I \times{ }_{\rho} M_{f}^{n}$. We point out that, to prove the following results, we do not require that the $f$-mean curvature $H_{f}$ of the spacelike hypersurface $\Sigma^{n}$ is constant.

Recall that a slab of a weighted GRW spacetime $-I \times_{\rho}$ $M_{f}^{n}$ is a region of the type

$$
\left[t_{1}, t_{2}\right] \times M_{f}^{n}=\left\{(t, p) \in-I \times_{\rho} M_{f}^{n}: t_{1} \leq t \leq t_{2}\right\} .
$$

Theorem 4. Let $\bar{M}^{n+1}=-I \times{ }_{\rho} M_{f}^{n}$ be a weighted GRW spacetime which obeys $(\log \rho)^{\prime \prime}(h) \leq 0$. Let $\psi: \Sigma^{n} \rightarrow \bar{M}^{n+1}$ be a complete spacelike hypersurface that lies in a slab of $\bar{M}^{n+1}$. If the $f$-mean curvature $H_{f}$ satisfies $H_{f}^{2} \leq\left(1 / \Theta^{2}\right)\left(\rho^{\prime}(h)^{2} / \rho(h)^{2}\right)$ and $|\nabla h| \in \mathscr{L}_{f}^{1}(\Sigma)$, then $\Sigma^{n}$ is a slice of $\bar{M}^{n+1}$.

Proof. From (10), we have

$$
\begin{aligned}
\frac{1}{\rho(h)} \Delta_{f} \rho(h)= & -n \frac{\rho^{\prime}(h)^{2}}{\rho(h)^{2}}+(\log \rho)^{\prime \prime}(h)|\nabla h|^{2} \\
& -n \frac{\rho^{\prime}(h)}{\rho(h)} H_{f} \Theta \\
\leq & -n \frac{\rho^{\prime}(h)^{2}}{\rho(h)^{2}}+(\log \rho)^{\prime \prime}(h)|\nabla h|^{2} \\
& +\frac{n}{2}\left(\frac{\rho^{\prime}(h)^{2}}{\rho(h)^{2}}+H_{f}^{2} \Theta^{2}\right) \\
\leq & \frac{n}{2}\left(H_{f}^{2} \Theta^{2}-\frac{\rho^{\prime}(h)^{2}}{\rho(h)^{2}}\right) \\
& +(\log \rho)^{\prime \prime}(h)|\nabla h|^{2} .
\end{aligned}
$$

By the hypotheses, we have $\Delta_{f} \rho(h) \leq 0$. Moreover, since $\Sigma^{n}$ lies in a slab, there is a positive constant $C$ such that

$$
|\nabla \rho(h)| \leq C \rho^{\prime}(h)|\nabla h| \in \mathscr{L}_{f}^{1}(\Sigma) .
$$

Therefore, we can apply Lemma 2 to get $\Delta_{f} \rho(h)=0$; that is, $\rho(h)$ is constant. Therefore $\Sigma^{n}$ is a slice.

Theorem 5. Let $\bar{M}^{n+1}=-I \times{ }_{\rho} M_{f}^{n}$ be a weighted GRW spacetime which obeys $\rho^{\prime \prime}(h) \leq 0$. Let $\psi: \Sigma^{n} \rightarrow \bar{M}^{n+1}$ be a complete spacelike hypersurface that lies in a slab of $\bar{M}^{n+1}$. If the $f$-mean curvature $H_{f}$ satisfies $H_{f}^{2} \leq \rho^{\prime}(h)^{2} / \rho(h)^{2}$ and $|\nabla h| \in \mathscr{L}_{f}^{1}(\Sigma)$, then $\Sigma^{n}$ is a slice of $\bar{M}^{n+1}$.

Proof. By a similar reasoning as in the proof of Theorem 4, we have

$$
\begin{aligned}
\frac{1}{\rho(h)} \Delta_{f} \rho(h) \leq & -n \frac{\rho^{\prime}(h)^{2}}{\rho(h)^{2}}+(\log \rho)^{\prime \prime}(h)|\nabla h|^{2} \\
& +\frac{n}{2}\left(\frac{\rho^{\prime}(h)^{2}}{\rho(h)^{2}}+H_{f}^{2} \Theta^{2}\right) \\
\leq & \frac{n}{2} H_{f}^{2} \Theta^{2}-\frac{n}{2} \frac{\rho^{\prime}(h)^{2}}{\rho(h)^{2}}+\frac{\rho^{\prime \prime}(h)}{\rho(h)}|\nabla h|^{2} \\
\leq & \frac{n}{2}\left(H_{f}^{2}-\frac{\rho^{\prime}(h)^{2}}{\rho(h)^{2}}\right) \Theta^{2} \\
& +\frac{\rho^{\prime \prime}(h)}{\rho(h)}|\nabla h|^{2},
\end{aligned}
$$

where the last inequality is due to $\Theta^{2} \geq 1$.

Taking into account the assumptions, we have $\Delta_{f} \rho(h) \leq$ 0 . Now in the same argument as in Theorem 4 , we have that $\Sigma^{n}$ is a slice.

Next, we will use the weak maximum principle to study the rigidity of the spacelike hypersurfaces in weighted GRW space-times.

Theorem 6. Let $\bar{M}^{n+1}=-I \times{ }_{\rho} M_{f}^{n}$ be a weighted GRW spacetime which satisfies $(\log \rho)^{\prime \prime} \leq 0$ and there is a point $h_{0} \in I$ such that $\rho^{\prime}\left(h_{0}\right)=0$. Let $\psi: \Sigma^{n} \rightarrow \bar{M}^{n+1}$ be a stochastically complete constant $f$-mean curvature $H_{f}$ spacelike hypersurface such that sup $h \geq h_{0}$, inf $h \leq h_{0}$ which is contained in a slab; then $\Sigma^{n}$ is $f$-maximal. In addition, if $\Sigma^{n}$ is complete and $|\nabla h| \in \mathscr{L}_{f}^{1}$, then $\sum^{n}$ is a slice.

Proof. We take the Gauss map $N$ of the hypersurface $\Sigma^{n}$ such that $\Theta>0$; from (7) we have $\Theta \geq 1$.

By Lemma 3, the weak maximum principle for the drifted Laplacian holds on $\Sigma^{n}$; then there exist two sequences $\left\{p_{j}\right\},\left\{q_{j}\right\} \subset \Sigma^{n}$ such that

$$
\lim _{j} h\left(p_{j}\right)=\inf h
$$




$$
\begin{aligned}
\lim _{j} \Delta_{f} h\left(p_{j}\right) \geq 0 . & \\
\lim _{j} h\left(q_{j}\right) & =\sup h, \\
\lim _{j} \Delta_{f} h\left(q_{j}\right) & \leq 0 .
\end{aligned}
$$

On the other hand, from (9), we have

$$
H_{f}=\frac{\left(-\rho^{\prime}(h) / \rho(h)\right)\left\{n+|\nabla h|^{2}\right\}-\Delta_{f} h}{n \Theta} .
$$

Since $\Sigma^{n}$ lies in a slab, if $h$ is bounded from below, then

$$
H_{f} \leq \frac{\left(-\rho^{\prime}(\inf h) / \rho(\inf h)\right)\left\{n+\left|\nabla h\left(p_{j}\right)\right|^{2}\right\}}{n \Theta\left(p_{j}\right)} .
$$

Moreover, if $h$ is bounded from above, we get

$$
H_{f} \geq \frac{\left(-\rho^{\prime}(\sup h) / \rho(\sup h)\right)\left\{n+\left|\nabla h\left(q_{j}\right)\right|^{2}\right\}}{n \Theta\left(q_{j}\right)} .
$$

Considering that the function $-\rho^{\prime} / \rho$ is increasing, then

$$
\begin{aligned}
H_{f} & \leq \frac{\left(-\rho^{\prime}(\inf h) / \rho(\inf h)\right)\left\{n+\left|\nabla h\left(p_{j}\right)\right|^{2}\right\}}{n \Theta\left(p_{j}\right)} \\
& \leq \frac{\left(-\rho^{\prime}\left(h_{0}\right) / \rho\left(h_{0}\right)\right)\left\{n+\left|\nabla h\left(p_{j}\right)\right|^{2}\right\}}{n \Theta\left(p_{j}\right)} \leq 0, \\
H_{f} & \geq \frac{\left(-\rho^{\prime}(\sup h) / \rho(\sup h)\right)\left\{n+\left|\nabla h\left(q_{j}\right)\right|^{2}\right\}}{n \Theta\left(q_{j}\right)} \\
\geq & \frac{\left(-\rho^{\prime}\left(h_{0}\right) / \rho\left(h_{0}\right)\right)\left\{n+\left|\nabla h\left(q_{j}\right)\right|^{2}\right\}}{n \Theta\left(q_{j}\right)} \geq 0 .
\end{aligned}
$$

Hence, $H_{f}=0$; that is, $\Sigma^{n}$ is a $f$-maximal spacelike hypersurface. Using (10), we have

$$
\Delta_{f} \rho(h)=-n \frac{\rho^{\prime}(h)^{2}}{\rho(h)}+|\nabla h|^{2} \rho(h)(\log \rho)^{\prime \prime}(h) \leq 0 .
$$

In the following, by the same argument as in Theorem 4, we have that $\Sigma^{n}$ is a slice.

\section{Weighted Static GRW Space-Times}

In this section, we obtain some rigidity results of stochastically complete hypersurfaces in weighted static GRW spacetimes $-I \times M_{f}^{n}$ by the weak maximal principle. Firstly, we give the following technical result which extended the corresponding conclusion in [12].
Lemma 7. Let $M$ be a stochastically complete Riemannian manifold and $u: M \rightarrow \mathbb{R}$ be a nonnegative smooth function on $M$. If there exists a positive constant $\lambda$ such that $\Delta_{f} u \geq \lambda u$, then $u=0$.

Theorem 8. Let $\psi: \Sigma^{n} \rightarrow-I \times M_{f}^{n}$ be a stochastically complete hypersurface with constant $f$-mean curvature $H_{f}$ in a weighted static GRW spacetime $-I \times M_{f}^{n}$. Assume that $K_{M} \geq$ $-k$ for some positive constant $k$ and the weighted function $f$ is convex. If $|\nabla h|^{2} \leq \alpha|A|^{2} /(n-1) k$ for some constant $0<\alpha<1$, then $\Sigma^{n}$ is a slice.

Proof. Let $E_{1}, \ldots, E_{n}$ be a (local) orthonormal frame in $\mathfrak{X}(\Sigma)$; using the Gauss equation, we have that

$$
\begin{aligned}
\operatorname{Ric}(X, X)= & \sum_{i=1}^{n}\left\langle\bar{R}\left(X, E_{i}\right) X, E_{i}\right\rangle+n H\langle A X, X\rangle \\
& +|A X|^{2},
\end{aligned}
$$

for $X \in \mathfrak{X}(\Sigma)$. Moreover, we also have

$$
\begin{aligned}
& \left\langle\bar{R}\left(X, E_{i}\right) X, E_{i}\right\rangle \\
& =K_{M}\left(X^{*}, E_{i}^{*}\right)\left(\left\langle X^{*}, X^{*}\right\rangle\left\langle E_{i}^{*}, E_{i}^{*}\right\rangle-\left\langle X^{*}, E_{i}^{*}\right\rangle^{2}\right),
\end{aligned}
$$

where $K_{M}$ is the sectional curvature of the fiber $M^{n}$ and $X^{*}=$ $X+\left\langle X, \partial_{t}\right\rangle \partial_{t}$ and $E_{i}^{*}=E_{i}+\left\langle E_{i}, \partial_{t}\right\rangle \partial_{t}$ are the projections of the tangent vector fields $X$ and $E_{i}$ onto $M^{n}$.

By a direct computation and considering the hypothesis $K_{M}>-k$, we get

$$
\begin{aligned}
& \sum_{i=1}^{n}\left\langle\bar{R}\left(X, E_{i}\right) X, E_{i}\right\rangle \\
& \quad \geq-k\left((n-1)|X|^{2}+(n-2)\langle X, \nabla h\rangle^{2}+|X|^{2}|\nabla h|^{2}\right) .
\end{aligned}
$$

Substituting (25) into (23),

$$
\begin{aligned}
\operatorname{Ric}(X, X) & \\
\geq & -k\left((n-1)|X|^{2}+(n-2)\langle X, \nabla h\rangle^{2}+|X|^{2}|\nabla h|^{2}\right) \\
& +n H\langle A X, X\rangle+|A X|^{2} .
\end{aligned}
$$

Furthermore, taking into account that the weighted function $f$ is convex, we have

$$
\text { Hess } \begin{aligned}
f(X, X) & =\overline{\operatorname{Hess}} f(X, X)-\langle\bar{\nabla} f, N\rangle\langle A X, X\rangle \\
& \geq-\langle\bar{\nabla} f, N\rangle\langle A X, X\rangle .
\end{aligned}
$$

Therefore,

$$
\begin{aligned}
\operatorname{Ric}_{f}(X, X) & \\
\geq & -k\left((n-1)|X|^{2}+(n-2)\langle X, \nabla h\rangle^{2}+|X|^{2}|\nabla h|^{2}\right) \\
& +n H_{f}\langle A X, X\rangle+|A X|^{2} .
\end{aligned}
$$


In particular, we have

$$
\begin{aligned}
\operatorname{Ric}_{f}(\nabla h, \nabla h) \geq & -k(n-1)|\nabla h|^{2}\left(1+|\nabla h|^{2}\right) \\
& +n H_{f}\langle A(\nabla h), \nabla h\rangle+|A(\nabla h)|^{2} .
\end{aligned}
$$
[2]):

Now we recall the Bochner-Lichnerowicz formula (see

$$
\begin{aligned}
\frac{1}{2} \Delta_{f}\left(|\nabla h|^{2}\right)= & |\operatorname{Hess} h|^{2}+\operatorname{Ric}_{f}(\nabla h, \nabla h) \\
& +\left\langle\nabla \Delta_{f} h, \nabla h\right) .
\end{aligned}
$$

From the fact that $H_{f}$ is a constant, we have

$$
\nabla \Delta_{f} h=-n H_{f} A(\nabla h) .
$$

By [14], we get

$$
\mid \text { Hess }\left.h\right|^{2}=|A|^{2}\left(1+|\nabla h|^{2}\right) .
$$

Using (29), (31), and (32) in (30), we have

$$
\frac{1}{2} \Delta_{f}|\nabla h|^{2} \geq\left(|A|^{2}-(n-1) k|\nabla h|^{2}\right)\left(1+|\nabla h|^{2}\right) .
$$

Finally, considering the hypothesis $|\nabla h|^{2} \leq \alpha|A|^{2} /(n-1) k$, we obtain

$$
\Delta_{f}|\nabla h|^{2} \geq 2(1-\alpha)|A|^{2}|\nabla h|^{2} .
$$

Thus, there is a positive constant $\lambda$ such that

$$
\Delta_{f}|\nabla h|^{2} \geq \lambda|\nabla h|^{2} .
$$

Therefore, $h$ is constant by Lemma 7 .

Theorem 9. Let $\psi: \Sigma^{n} \rightarrow-I \times M_{f}^{n}$ be a stochastically complete hypersurface with constant $f$-mean curvature $H_{f}$ in a weighted static GRW space-time $-I \times M_{f}^{n}$. Assume that the sectional curvature $K_{M}$ is nonnegative and the weighted function $f$ is convex. If $|\nabla h|^{2}$ is bounded from above, then $\Sigma^{n}$ is $f$-maximal.

Proof. As in the proof of Theorem 8, taking into account that the hypothesis $K_{M}$ is nonnegative, there is a constant $k \geq 0$ such that

$$
\frac{1}{2} \Delta_{f}|\nabla h|^{2} \geq|\operatorname{Hess} h|^{2}+(n-1) k|\nabla h|^{2}\left(1+|\nabla h|^{2}\right) .
$$

Moreover, considering the relation $n \mid$ Hess $\left.h\right|^{2} \geq\left(\Delta_{f} h\right)^{2}$, we have

$$
\Delta_{f}|\nabla h|^{2} \geq \frac{2}{n}\left(\Delta_{f} h\right)^{2} .
$$

Using (9) and (37), we obtain

$$
\Delta_{f}|\nabla h|^{2} \geq 2 n H_{f}^{2} \Theta^{2} \geq 2 n H_{f}^{2} \geq 0 .
$$

By the hypothesis that $|\nabla h|^{2}$ is bounded from above, applying Lemma 3, the weak maximum principle, we get

$$
0 \geq \lim _{k} \Delta_{f}|\nabla h|^{2}\left(q_{k}\right) \geq 2 n H_{f}^{2} \geq 0 .
$$

Therefore $\Sigma^{n}$ is $f$-maximal.
As a consequence of the proof of Theorem 8, we can get the following corollary.

Corollary 10. Let $\psi: \Sigma^{n} \rightarrow-I \times M_{f}^{n}$ be a stochastically complete hypersurface with constant $f$-mean curvature $H_{f}$ in a weighted static GRW space-time $-I \times M_{f}^{n}$. Assume that $K_{M} \geq$ $-k$ and $\overline{\text { Hess }} f \geq-\beta$ for some positive constants $k$ and $\beta$. If $|\nabla h|^{2} \leq \alpha|A|^{2} /((n-1) k+\beta)$ for some constant $0<\alpha<1$, then $\Sigma^{n}$ is a slice.

\section{Conflicts of Interest}

The authors declare that they have no conflicts of interest.

\section{Acknowledgments}

This work is supported by National Natural Science Foundation of China (no. 11371076).

\section{References}

[1] D. Bakry and M. Mery, "Diffusions hypercontractives," in Sminaire de probabilits, XIX, vol. 1123 of Lecture Notes in Math., pp. 177-206, Springer, Berlin, Germany, 1983.

[2] G. Wei and W. Wylie, "Comparison geometry for the BakryEmery Ricci tensor," Journal of Differential Geometry, vol. 83, no. 2, pp. 377-405, 2009.

[3] M. P. Cavalcante, H. F. de Lima, and M. S. Santos, "On Bernstein-type properties of complete hypersurfaces in weighted warped products," Annali di Matematica Pura ed Applicata. Series IV, vol. 195, no. 2, pp. 309-322, 2016.

[4] M. P. Cavalcante, H. F. de Lima, and M. S. Santos, "New CalabiBernstein type results in weighted generalized RobertsonWalker spacetimes," Acta Mathematica Hungarica, vol. 145, no. 2, pp. 440-454, 2015.

[5] H. F. de Lima, A. M. Oliveira, and M. S. Santos, "Rigidity of complete spacelike hypersurfaces with constant weighted mean curvature," Beiträge zur Algebra und Geometrie/Contributions to Algebra and Geometry, vol. 57, no. 3, pp. 623-635, 2016.

[6] B. O'Neill, Semi-Riemannian Geometry with Applications to Relativity, Academic Press, San Diego, Calif, USA, 1983.

[7] L. J. Alas, A. Romero, and M. Sánchez, "Uniqueness of complete spacelike hypersurfaces of constant mean curvature in generalized Robertson-Walker spacetimes," General Relativity and Gravitation, vol. 27, no. 1, pp. 71-84, 1995.

[8] M. Gromov, "Isoperimetry of waists and concentration of maps," Geometric and Functional Analysis, vol. 13, no. 1, pp. 178215, 2003.

[9] J. S. Case, "Singularity theorems and the Lorentzian splitting theorem for the Bakry-Emery-Ricci tensor," Journal of Geometry and Physics, vol. 60, no. 3, pp. 477-490, 2010.

[10] A. Caminha, "The geometry of closed conformal vector fields on Riemannian spaces," Bulletin of the Brazilian Mathematical Society. New Series. Boletim da Sociedade Brasileira de Matemática, vol. 42, no. 2, pp. 277-300, 2011.

[11] S. T. Yau, "Some function-theoretic properties of complete Riemannian manifold and their applications to geometry," Indiana University Mathematics Journal, vol. 25, no. 7, pp. 659-670, 1976.

[12] S. Pigola, M. Rigoli, and A. G. Setti, "A remark on the maximum principle and stochastic completeness," Proceedings of the 
American Mathematical Society, vol. 131, no. 4, pp. 1283-1288, 2003.

[13] M. Rimoldi, Rigidity Results for Lichnerowicz Bakry-Émery Ricci Tensors [Ph.D. thesis], Università degli Studi di Milano, Milano, Italy, 2011.

[14] J. M. Latorre and A. Romero, "Uniqueness of noncompact spacelike hypersurfaces of constant mean curvature in generalized Robertson-Walker spacetimes," Geometriae Dedicata, vol. 93, pp. 1-10, 2002. 


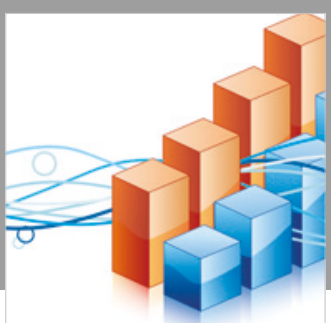

Advances in

Operations Research

\section{-n-m}
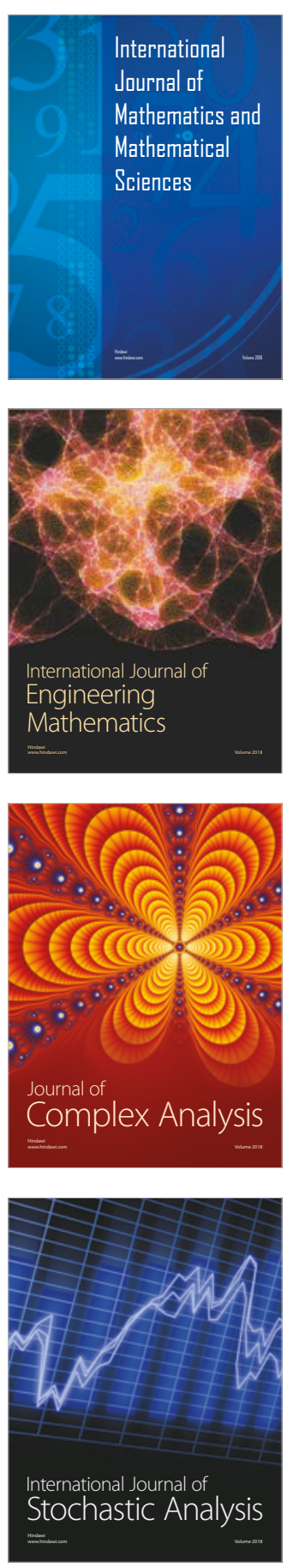
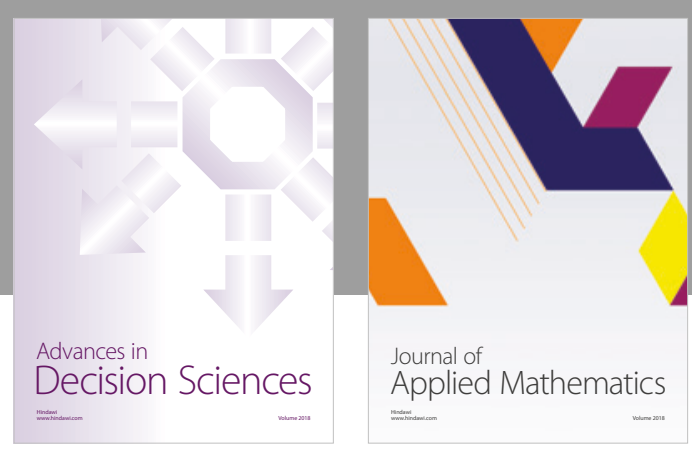

Journal of

Applied Mathematics
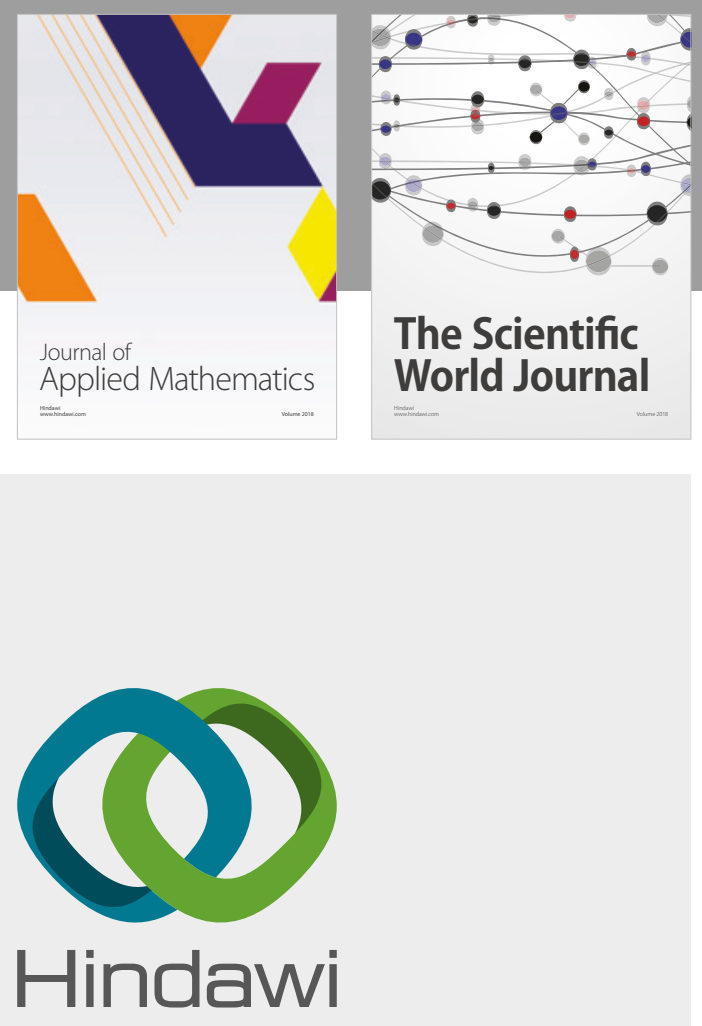

Submit your manuscripts at

www.hindawi.com

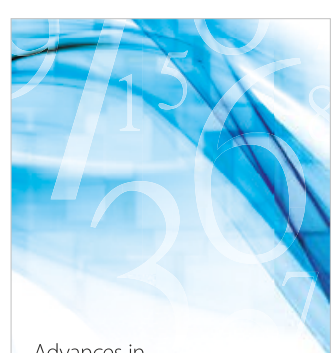

Advances in
Numerical Analysis
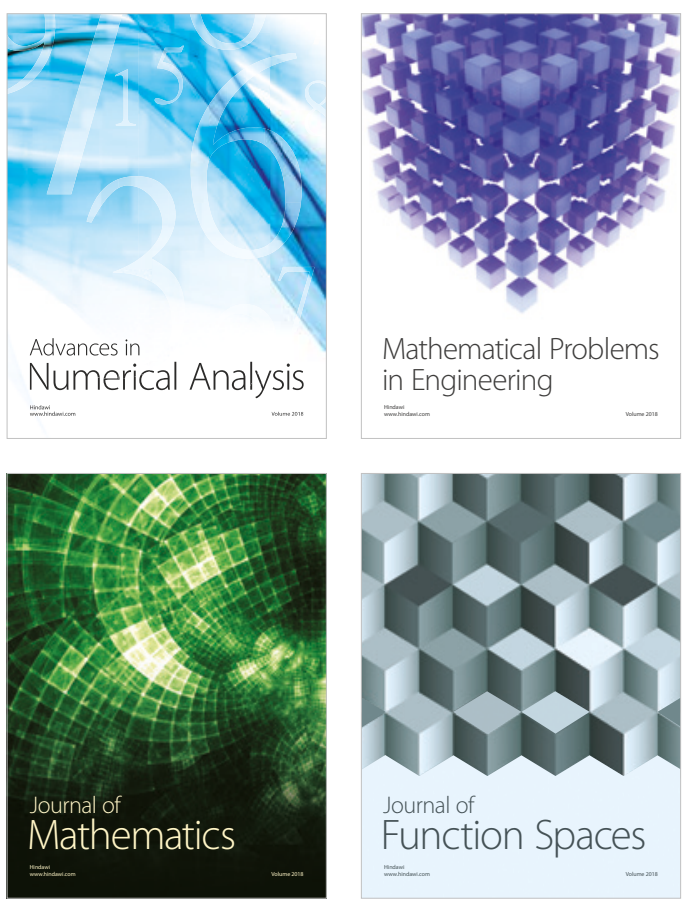

Mathematical Problems in Engineering

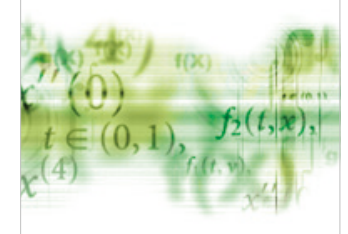

International Journal of

Differential Equations

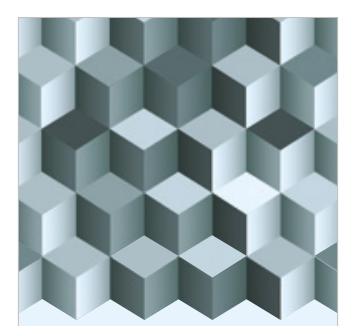

Journal of

Function Spaces

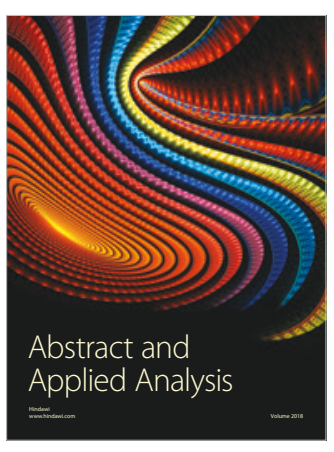

The Scientific

World Journal

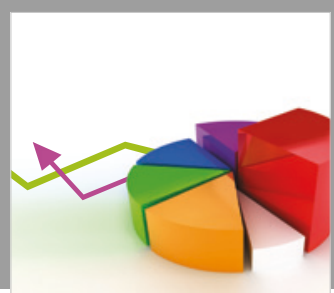

Journal of

Probability and Statistics
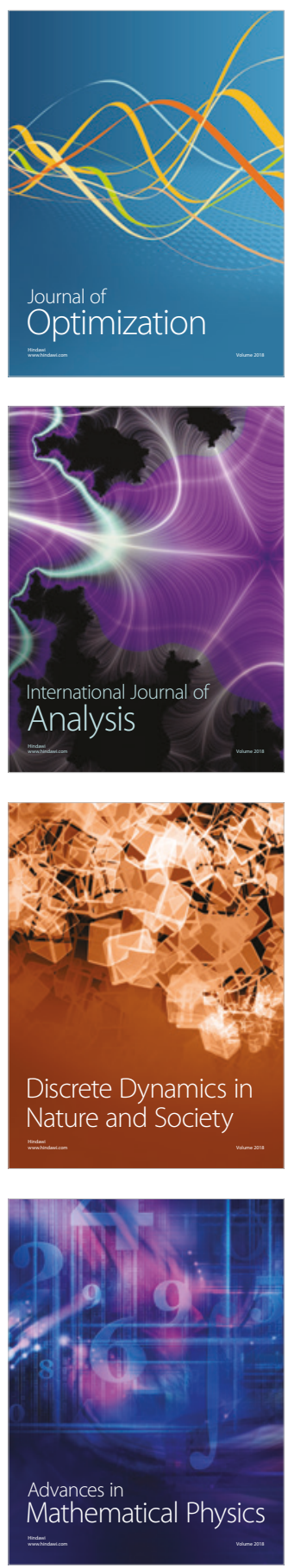